\title{
La Manekine grecque et sa source italienne.
}

En notant que la légende qui fait le sujet du roman français La Manekine se trouve aussi dans la littérature grecque et notamment dans le livre intitulé ' $H \tau \tilde{\omega} \nu \dot{\alpha} \mu \alpha \rho \tau \omega \lambda \tilde{\omega} \nu \sigma \omega \tau \eta \rho i \alpha(1641)$ du moine Agapios Landos de Crète ${ }^{1}$ ), M. Krumbacher (Gesch. der byz. Lit. 869) ajoute que personne n'a expliqué le rapport généalogique de ces deux textes.

Je ne veux pas, moi non plus, expliquer le rapport très compliqué entre la Manekine et le conte qui se trouve dans le lirre d'Agapios: ce que, pour le moment, je voudrais bien essayer, c'est d'examiner le rapport plus direct entre le conte d'Agapios et un autre, italien, qui se trouve dans un recueil analogue de miracles, les Miraculi de la gloriosa verzene Maria (1475), et qui a pour titre Come la gloriosa verzene Maria campa da molte insidie una fiola de uno imperatore ala quale li era state tagliate le mane. ${ }^{2}$ ) On a déjà signalé qu'il y a du rapport entre ces deux oeuvres, l'italienne et la grecque, et même on a établi que le conte grec dérive de l'italien; cependant, comme on va le voir tout de suite, la chose a été traitée quelque peu sommairement, et l'on n'est pas au clair si, de la part d'Agapios, il s'agit d'une imitation seulement ou birn d'une véritable traduction.

Voici, comme preuve à ce que nous venons de dire, ce que, autant que je sache, on a écrit jusqu' à maintenant sur les sources de la Manekine grecque. - Le premier qui a recherché ces sources est I. V. Leclerc, qui a remarqué que le sujet qui se trouve dans le conte

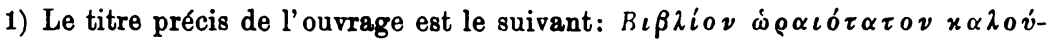

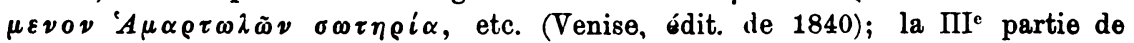
l'ouvrage contient les miracles de la Vierge Marie: 'Ex $\tau \tilde{\omega} \nu$ v $\pi \varepsilon \rho q v \tilde{\omega} \nu \boldsymbol{v} \alpha v$ -

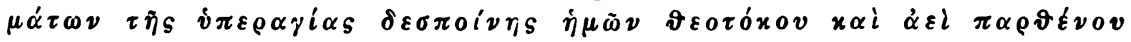

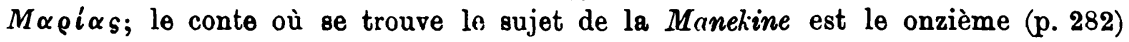

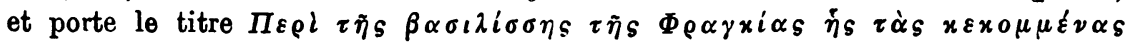

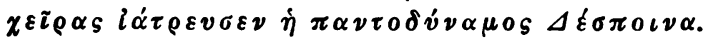

2) Dans l'édit. de 1855, Urbino (Li Miracoli della Madonna), dont nous nous servons plus loin, le conte est le dixième du recueil (p. 20); dans l'édit. de 1475, Vicenza, dont nous venons de citer le titre, il est le onzième. 
d'Agapios est traité dans la Manekine; la lettre où il fait part de cette remarque a été communiquée par M. A. Wesselofsky (Novella della figlia del re di Dacia, Pisa, 1866, chap. III), qui, lui aussi, a étudié, comme on sait, la célèbre légende de la Fille sans mains. En s'appuyant sur l'idée de Leclerc, M. Ch. Gidel (Etudes sur la litt. grecque moderne; 1866, p. 289-301) a comparé le conte d'Agapios avec le vieux roman français et $\mathrm{y}$ a trouvé "la plus grande ressemblance“. - M. A. D'Ancona (Sacre rappres. dei sec. XIV, XV e XVI, 1872, vol. III; introd. à la Rappr. di Stella, p. 317-319) vient à combattre l'idée de M. M. Leclerc et Gidel, et se prononce pour les Miraculi de la gloriosa verzene Maria comme source du conte grec. „Dal testo italiano dei Miraculi deriva, secondo noi, la narrazione di Agapio di Creta che il Gidel fa invece derivare dal romanzo francese della Manekine; chiunque confronti la versione greca colla leggenda italiana e col poema francese, si avredrà dell'errore in che è caduto il Gidel" - c'est ainsi que s'exprime M. D'Ancona, sans cependant nous faire voir plus directement dans les deux textes. - M. E. Legrand (Recueil de contes pop. grecs, 1881, préface), en parlant du conte d'Agapios, qu'il insère dans son recueil (Nr. 28: La belle sans mains), et ne paraissant pas connaître ce que, sur le sujet, ont écrit M. M. Gidel et D'Ancona, dit: „Notre conte rappelle la Manekine, mais il ne saurait en découler directement, car il est inadmissible qu'Agapios ait pu connaître ce vieux roman; il est vraisemblable qu'il avait entre les mains quelque imitation italienne de la Manekine, et qu'il s'en est servi pour rédiger sa pieuse élucubration". - M. Th. de Puymaigre (La fille aux mains coupées, Revue de l'hist. des relig. X, 1884, p. 193-209), en développant l'idée de M. Legrand, se souvient des recherches de M. D'Ancona et dit p. 203: „Legrand ... udmet que le moine (Agapios) dut connaitre quelque imitation italienne de ce roman (Manekine); cela parait certain: D'Ancona parle d'un vieux roman, Miracoli della Madonna ..., le moine grec dut avoir entre les mains ce livre de Miracoli“. - M. H. Suchier (Oeuvres poétiques de Ph. de Reni sire de Beaumanoir, 1884, I, introd. p. L), enfin, en parlant du conte italien des Miraculi, dit simplement: "une traduction grecque de ce miracle se trouve dans le Salut des

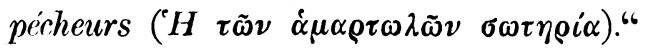

J'ai donc cru qu'une petite analyse du conte d'Agapios et de celui des Miraculi ne saurait être superflue pour notre question, et que, peut-être, en comparant les deux textes, on pourrait voir quelque chose de pius.

Or, quel est le rapport entre le conte grec et l'italien?

D'abord, le conte d'Agapios n'est pas une „traduction“, et c'esi 
évident pour quiconque a eu les deux textes entre les mains. Le conte d'Agapios n'est véritablement qu'une imitation, et une imitation qui suit de près son original et qui en contient tous les principaux moments et dans le même ordre. ${ }^{1}$ ) Dans les deux contes, un empereur de France, étant demeuré veuf avec une fille, se remarie; sa seconde femme, qui était d'une très grande beauté, se met tout de suite à haïr sa belle-fille, parce que celle-ci était encore plus belle. Profitant d'une absence de son mari, la nouvelle impératrice donne ordre de tuer la jeune fille et de lui couper les mains comme preuve du crime accompli. L'ordre n'est exécuté qu'à demi, et les serviteurs qui en étaient chargés, appitoyés par les pleurs et les prières de la malheureuse, se contentent de lui couper les mains et de la laisser au désert. La jeune fille n'y reste pas longtemps; le fils d'un duc la trouve, la conduit chez lui, et, quelque temps après, la prend pour épouse. Vient ensuite, de la part de l'empereur, une invitation du duc à un tournoi; le jeune duc s'y rend, son père étant vieux et faible. Pendant l'absence du jeune duc, sa femme accouche de deux enfants; on envoie immédiatement un courrier pour annoncer la bonne nouvelle au jeune père, et celui-ci répond par une lettre où il recommanda chaleureusement sa femme à son père. Cette lettre, l'impératrice (informée déjà que la femme du jeune duc n'était autre que sa belle-fille) la prend, en enivrant le courrier qui la portait, et en substitue une autre (ou change la première) où elle donna ordre de tuer la jeune femme avec ses enfants. De nouveau, la vie est sauvée à l'héroïne, que, pour toute punition, le vieux duc fait conduire au désert avec les deux enfants. Au désert, la malheureuse est recueillie par un ermite; la Vierge Marie, à laquelle elle était trèsdévouée et qu'elle ne cessait de prier, lui apparaît bientôt et lui restitue les mains. Le jeune duc, étant de retour et apprenaut ce qu'est devenue sa femme, se met tout de suite à la chercher, la trouve bientôt et la conduit chez lui; alors elle fait connaître sa naissance, soigneusement cachée jusque-là. On se rend auprès de l'empereur, qui reçoit arec joie sa fille et son gendre et fait brâler la méchante marâtre.

L'analogie de ces deux contes n'a pas besoin d'être analysée d'une manière plus détaillée, tant elle est évidente; aussi avons-nous dans cette courte analyse, que nous avons fait moins pour prouver cette analogie que pour donner un résumé de nos contes, omis bien des détails qui les rapprochent encore plus. Ce qui est important ici, ce

1) L'ordre n'est interrompu qu'une seule fois. Dans le conte d'Agapios, le jeune duc revient du tournoi avant que la Vierge Marie restitue les mains à l'héroïne; dans le conte des Miraculi, cels arrive immédiatement après. 
ne sont pas les analogies mais les différences des deux contes; et ces différences sont les suivantes.

D'abord, le conte d'Agapios est plus long, et plus amplifié que le conte italien. Ce qui dans le conte italien n'est qu'indiqué, dans le conte grec est bien des fois traduit en une scène entière; si dans le premier p. ex. on mentionne que tels personnages tenaient conseil ou se disaient quelque chose, dans le second tout ce qu'on y disait est souvent exprimé in extenso, sous forme de dialogue. Le conte italien, p. ex. n'a pas un mot de ce que l'héroïne disait aux serviteurs qui devaient la tuer et lui couper les mains, et se contente de dire qu'elle „con grandissimi pianti si raccomandava alla gl. V. Maria“; le conte grec, au contraire, y a un dialogue complet entre elle et les serviteurs, et assez long; etc. Outre les dialogues, le conte d'Agapios a aussi des amplifications d'autre espèce; les descriptions y sont quelquefois plus détaillées et les choses sont racontées avec plus d'ampleur. Le conte italien, p. ex., raconte simplement comment le jeune duc trouva sa femme, et ses enfants (,pervenne alla spelonca ... dov'era la detta giovane, e trovandola..."); le conte grec, cependant, y met plus de relief et sait raconter comment la jeune duchesse, „le jour étant paru, entendit des voix d'hommes, et, en sortant, vit son mari" etc.

Mais, outre ces amplifications, le conte d'Agapios a aussi quelquefois des détails et des motifs qui ne se trouvent pas dans le conte italien. Ces motifs, les voici. Dans le conte grec, l'héroüne a son nom, Marie; dans l'italien, elle n'est désignée que par des expressions: la giovane, la detta giovane, etc. Lorsque l'impératrice cherche à séduire le serviteur à tuer la jeune fille, elle lui promet, dans le conte grec, de le récompenser fort bien et de le faire le premier de l'empire („xai

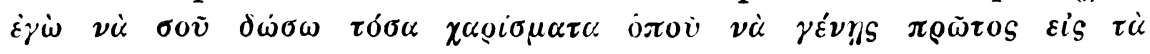
$\beta \omega \sigma i \lambda \varepsilon \iota \alpha^{\prime \prime}$, lui dit-elle); dans le conte italien, elle n'appelle qu'à la fidélité des serviteurs („e allora conoscerò certamente essere miei fedeli servi“, leur dit-eile). Dans la même occasion, l'impératrice dénigre sa

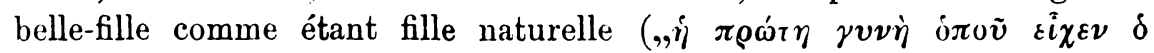

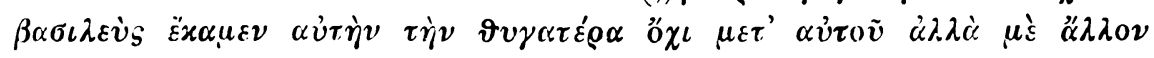

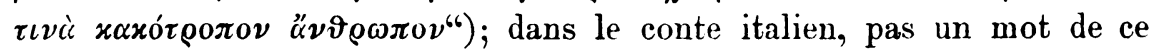
dénigrement. L'impératrice, d'ailleurs, avant de chasser sa belle-fille au désert, la trompe en lui disant qu'elle l'envoie se promener dans

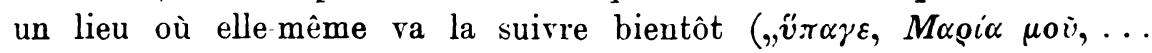

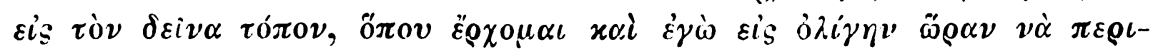
$\delta\left\llcorner\alpha\left(\alpha_{c} \sigma 0 \mu \varepsilon v^{*}\right)\right.$; dans le conte italien, la marâtre ne voit même pas la jeune fille à la veille du supplice. Dans le conte grec, c'est à la chasse

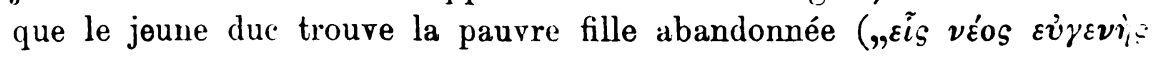




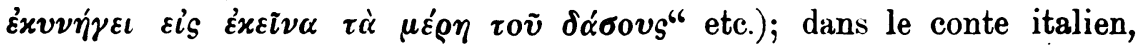
cela arrive pendant un simple passage à travers le désert („un figliuolo di un duca passando per quel deserto, etc"). Lorsque l'empereur, étant de retour, demande sa fille, l'impératrice lui répond qu'elle s'est enfuie

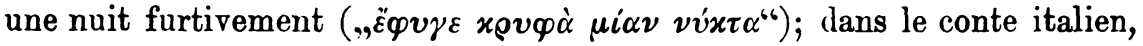
cette explication n'est pas donnée. En enivrant le courrier et en prenant la lettre du jeune duc, l'impératrice, d'après le conte grec,

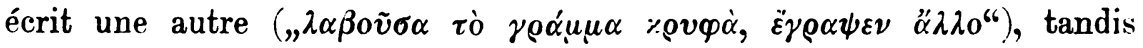
que, d'après le conte italien, elle ne fait qu'effacer quelques mots dans la lettre du duc et écrire d'autres mots (,e in questo luogo dove diceva, della mia donna e de' miei figliuolini buona cura, rase sottilmente, e scrisse cosi, etc"). L'ermite, en voyant pour la première fois l'héroïne

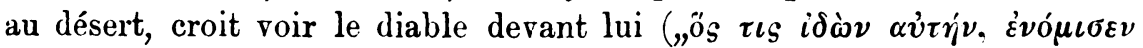

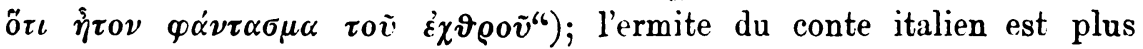
calme et n'y voit rien de surnaturel (,,vedendola così abbandonata, con que' due figliuolini in braccio, si commosse a grande pietà). Etc.

Il est intéressant de remarquer que tous ces motifs que nous venons d'énumérer et qui se trouvent dans le conte d'Agapios et pas dans celui des Miraculi, se trourent aussi dans un autre ouvrage italien qui dérive de celui-ci; je veux nommer Stella, drame religieux du siècle XV ou XVI. Dans ce drame aussi, l'héroïne a son nom (Stella); l'impératrice fait aussi des promesses à des serviteurs („farò voi grandi ed alti nel mio regno", leur dit-elle) et dénigre sa belle-fille de la même manière que dans le conte grec (,del mio sposo imperier la falsa nata“, dit-elle en parlant de la jeune fille). Là aussi, la jeune fille est trompée sur fe but de voyage qu'on lui fait entreprendre, et les serviteurs lui disent qu'on la conduit „incontro al padre tuo ch'oggi s'aspetta"; là aussi, c'est à la chasse que le jeune duc trouve l'héroïne (v. la scène entière où le jeune homme prie son père de lui permettre d'aller à la chasse); là aussi, l'impératrice répond à ,l'imperadore" qui demande „or dov'è Stella mia?“ qu'un matin on ne la vit plus. La lettre du jeune duc est aussi remplacée par une autre: „la regina... gli (au courrier) toglie la lettera e pouvenne un'altra contrafatta". L'ermite, en voyant la paurre sans mains, fait le signe de la croix et dit: „Domin, che cosa mostruosa fia!“1)

Est-il permis de croire que le vieux moine grec s'est inspiré du drame aussi bien que du conte italien pour écrire le sien? Il a peut-

1) N'ayant pas pour le moment le texte de la Stella entre les mains, et ne me servant que de notes que j'ai faites pendant la lecture de ce drame à la Bibliothèque de l'Université de Vienne en 1903, je ne puis continuer la liste des analogies du conto des Miraculi et du drame de Stella. 
être pu voir une représentation de Stella à Venise, où l'on peut supposer qu'il habita quelque temps et assez longtemps, puisque son livre a été imprimé dans cette ville italienne et n'a paru qu'en 1641 bien qu'il fât achevé en 1639, comme on le voit dans un manuscrit de son ouvrage qui se trouve au Mont Athos. (Voir Sp. Lambros, Catalogue of the greek mss. on Mount Athos, 1900, II vol. Nr. 4462).

Belgrade.

Pavle Popović.

\section{La biographie de Kasia dans la littérature serbe.}

Dans un roman de Milovan Vidaković (romancier serbe, 1780 1841), intitulé Selim et Merima (1839), il y a un passage (p. 45, 46) qui renferme la biographie de Kasia la poétesse (cf. Krụmbacher, Gesch. der byz. Lit. ${ }^{2}$ S. 715), brièvement racontée, et qui représente, autant que je sache, la seule trace de cette biographie dans la littérature serbe. Voici ce passage: - «Ma gouvernante, Grecque d'origine, - dit Merima, héroïne du roman - m'a conté l'histoire de Kasia. Il y avait autrefois, disait-elle, un empereur grec qui était jeune et voulait se marier. Pour pouvoir trouver celle qu'il choisirait pour épouse, il fit venir dans son palais Kasia et une autre jeune fille. Toutes les deux étant extrêmement belles, l'empereur, en les regardant, ne put décider laquelle des deux épouser; alors il leur posa certaines questions afin de connaître, par les réponses, leur cœur et leur intelligence. Les réponses faites, l'empereur choisit l'autre, et laissa partir Kasia. Celle ci lui dit alors: « «Sire, puisque je n'ai pas le bonbeur extrême de devenir votre épouse, je ne serai l'épouse de personne.»» En disant ces mots, elle alla dans un couvent, se fit religieuse et passa toute sa vie à servir Dieu et chanter sa gloire. Elle a composé, dit-on, plusieurs cantiques et hymnes qu'on chante encore dans les églises."

Belgrade.

P. Popović. 